\title{
On the analysis of viability data: an example with Drosophila
}

\author{
J. C. Adell, \\ A. Moya, \\ V. Molina and \\ F. González-Candelas
}

Departament de Genètica, Universitat de València, c/Dr Moliner, 50 Burjassot, E-46100 Valencia, Spain.

Larval competition experiments involving two wild type and eight mutant strains of Drosophila melanogaster have been carried out following the substitution procedure proposed by Mather and Caligari (1981). Our main goal has been to compare the competitive abilities of two phenotypically indistinguishable strains (wild and Oregon-R) by means of their responses with eight different mutants. Prior to the analyses of viability data, we have studied the normalizing effect of several transformations in order to determine which was best suited for the analyses. The differences found among the five transformations tested and the untransformed data were not very great. The folded power transformation (Mosteller and Tukey, 1977) was finally chosen.

No constant pattern in the responses of the two wild type strains to the mutant competitors was detected. This leads us to conclude that the nature of the competition between the two wild type strains cannot be predicted from a knowledge of their competition with other strains.

\section{INTRODUCTION}

Interactions that occur among individuals belonging to the same species under competitive conditions can be classified as intragenotypic and intergenotypic. The development of the theoretical basis for the study of these phenomena has been made by Mather and Caligari (Caligari, 1980; Mather and Caligari, 1981, 1983; Caligari and Mather, 1984, 1988). The effects of different genotypes on each other are studied from the simplest model (monocultures) with only one genotype present, to cases with increasing complexity (duo- and triocultures) with two and three different genotypes simultaneously present in the same culture.

The first step in their method consists of the detection and separation of intragenotypic from intergenotypic interactions, which enables their comparison. Intragenotypic interactions are estimated from monocultures (Caligari, 1980), by way of the linear dependence of larva-to-adult viability on density.

Once intragenotypic interactions had been studied inter-genotypic interactions are estimated from duocultures using a substitution design (Mather and Caligari, 1981) which involves the replacement of a given number of individuals of the indicator strain by exactly the same amount of individuals from the competing one. Hence, total culture density is kept constant.

Competitive responses are analysed by comparison of the linear regression slopes of monoand duocultures (see fig. 1 in Mather and Caligari, 1981). This allows the classification of competitive interactions into competition, hypercompetition, facilitation, indifference, and equality between intra- and intergenotypic interactions.

However several problems, both biological and statistical, are raised by this method. The main biological problem is related to the determination of intergenotypic interactions between different genotypes with undistinguishable phenotypes. Eggleston (1987), working with Drosophila melanogaster proposed the use of the mutant $y^{2}$ as a marker, because the competitive response of a wild strain when carrying that marker was shown to be the same as that without it. But this solution requires the introduction of the marker into the genome of interest, with the corresponding disruption, and also assumes that this marker interacts in exactly the same way with any other genome.

The statistical problems arise from the requirement for normality of errors, additivity of structure 
and homogeneity of variances (Atkinson, 1985) in every least-squares regression. Several different transformations have been proposed to achieve these requirements. Many of them belong to a general family, known as the power family of transformations (Box and Cox, 1964). Among these, the most commonly used are the logarithmic and the square root transformations and in fact, many analyses of larval competition make use of one or the other (Mather and Caligari, 1981; Mather et al., 1982; Kelley, 1985; Kelley and Clay, 1987). Another possible transformation, proposed originally by Anscombe (1948) and used subsequently by Mather and Caligari (1981) and Caligari and Mather (1988), is the angular transformation, which does not belong to the power family.

The power transformations are appropriate for non-negative responses, but they may not be so when there is an upper limit for the dependent variable, such as we find in analyses of viability (Atkinson, 1985). Several other general transformations have been proposed to solve this problem.

In the experiments described here we have studied the folded power transformation (Mosteller and Tukey, 1977), the transformation proposed by Aranda-Ordaz (1981), and the angular and the power transformations in order to determine whether they lead to different statistical and biological conclusions and to find out which of them better approaches the requirements of normality, additivity and homoscedasticity.

\section{MATERIAL AND METHODS}

\section{Experimental design}

In these experiments, ten different strains of Drosophila melanogaster were used. Half of them were captured in a cellar near Requena (Valencia, Spain) and the rest were laboratory strains. Each group consisted of one wild and four eye colour mutant strains. The cellar strains were named wild, 1.51.3, 2.54.A (identified as $c d^{770}$ ), 2.58.A (identified as $s e^{790}$ ) and 2.74. B. The laboratory strains were Oregon-R, se, bo, $v$ and $w$, and were supplied by the Drosophila Collection Center at Bowling Green (Ohio, U.S.A.).

All these strains were kept in the laboratory in half-pint bottles supplied with Lewis' medium at $25^{\circ} \mathrm{C}$ and 60 per cent relative humidity. The experiments were carried out with newly hatched larvae $( \pm 2 \mathrm{~h})$ seeded in $50 \times 8 \mathrm{~mm}$ vials with $0.5 \mathrm{ml}$ of food. Each vial contained $50 \mathrm{mg}$ of yeast. This implies that the amount of yeast per larva $(0.71 \mathrm{mg})$ is not enough for all the larvae in a vial to develop into adults (Economos et al., 1982).

Monocultures for all strains were initiated with the following number of larvae: $70,66,60,35,10$ and 4 . In addition, wild type monocultures with 50 larvae and mutant monocultures with 20 larvae were included. Each series therefore consisted of seven different initial densities. Duocultures were performed according to the substitution design (Mather and Caligari, 1981), and each wild type strain was set to compete with all eight mutant strains. The initial numbers of wild and mutant larvae were: $66 / 4,60 / 10,50 / 20,35 / 35,10 / 60$, $4 / 66$, respectively. In all cases 20 replicates were initiated although not all of these survived in every case. All of the replicates for each monoculture or duoculture were raised simultaneously, but the different competition systems were spaced over several months.

\section{Statistical transformations}

The family of power transformations was proposed by Box and Cox (1964) and it belongs to the more general class of parametric transformations:

$$
y(\lambda)= \begin{cases}\left(y^{\lambda}-1\right) / \lambda & (\lambda \neq 0) \\ \log y & (\lambda=0)\end{cases}
$$

where $y(\lambda)$ is the transformed variable and $\lambda$ the corresponding parameter.

A problem that arises with the use of parametric transformations on the same variable is to decide which value of the parameter conforms better to our requirements. As the use of different $\lambda$ involves changes in scale, it is not possible to compare directly their residual sums of squares. The solution proposed by Box and Cox (1964) is to perform the comparison by means of the corresponding log-likelihood

$$
L_{\max }(\lambda)=-(n / 2) \log \{R(\lambda) / n\}
$$

where $n$ is the number of observations and $R(\lambda)$ is the residual sum of squares of the normalized transformation (Atkinson, 1985).

Two other transformations used in this analysis are also parametric. The folded power transformation (Mosteller and Tukey, 1977) and the one proposed by Aranda-Ordaz (1981) are designed to overcome the difficulties found in the application of the power transformation to data with an upper limit (Atkinson, 1985). In this paper we analyze larva-to-adult viabilities which, obviously, belong to the interval $[0,1]$. The choice of parameters is made as in the previous case by comparing the log likelihoods. 
The folded power transformation is expressed as

$$
u(\lambda)= \begin{cases}y^{\lambda}-(1-y)^{\lambda} & (\lambda \neq 0) \\ \log \{y /(1-y)\} & (\lambda=0)\end{cases}
$$

for $0 \leqq y \leqq 1$. For values of $y$ near zero the transformation behaves like the power transformation $y^{\lambda}$, whereas for $y$ near one it behaves like $(1-y)^{\lambda}$ (Atkinson, 1985).

The Aranda-Ordaz proposal (1981) was designed originally for the analysis of binary data, and is expressed as:

$$
u_{\mathrm{A} 0}(\lambda)= \begin{cases}{\left[y^{\lambda}-(1-y)^{\lambda}\right] /\left[y^{\lambda}+(1-y)^{\lambda}\right]} & (\lambda \neq 0) \\ \log \{y /(1-y)\} & (\lambda=0)\end{cases}
$$

The normalized expressions for all of these transformations can be found in Atkinson (1985).

For the three parametric transformations, likelihoods were computed for values of $\lambda$ from -2 to +2 , in $0 \cdot 1$ increments. Optimal values for $\lambda$ were estimated using the residual sums of squares following analyses of variance of viability data. Confidence intervals ( $P \geqq 0.95$ ) for the parameters were estimated in each case, and those values that appeared most frequently were chosen as the $\lambda$ parameter for each transformation.

The angular transformation (Anscombe, 1948) is not parametric and it has been applied extensively to the analysis of viability data (Moya and Botella, 1985; Moya and Castro, 1986; Molina et al., 1988; Caligari and Mather, 1988, among others). This transformation is given by

$$
w(y)=\arcsin \sqrt{y} \quad(0 \leqq y \leqq 1) .
$$

Analyses which utilised these four transformations, as well as the square root transformation, were compared with the results obtained using untransformed data. Least squares linear regressions for mono- and duocultures together with the necessary analyses of covariance were performed in order to compare the slopes obtained in pure and mixed cultures.

\section{RESULTS}

Due to the large volume of raw data obtained in this experiment, we present only the subsequent analyses. All raw data are available from the authors upon request.

The first step in our analysis involved the determination of $\lambda$ for the three parametric transformations. Analyses of variance of viabilities with respect to initial densities were carried out for 41 different values of $\lambda$, in the interval $[-2 \cdot 0,2 \cdot 0]$ with $0 \cdot 1$ increments, for each normalized transformation. The corresponding likelihoods were computed according to equation (2) and 95 per cent confidence intervals were estimated. A total of 5166 ANOVA were made (three transformations $\times 41$ parameter values $\times 42$ cultures) using procedure ONEWAY of the $\mathrm{SPSS}^{+} / \mathrm{PC}$ statistical package with an AT-like microcomputer.

In some cases the value of $\lambda$ corresponding to the maximum likelihood was beyond our initial limits and, therefore, it was not possible to determine their confidence intervals so they were not taken into account in the determination of the optimal $\lambda$.

A plot of the number of cases in which a given value of $\lambda$ belongs to the 95 per cent confidence interval is shown in fig. 1. Determination of the optimal $\lambda$ value has to be based not only on maximized likelihood but also on the requirement of easy biological interpretation (Box and Cox, 1964). Accordingly, for the power transformation we decided to choose $\lambda=0.0$ instead of $\lambda=-0.5$ because their number of appearances is almost equal ( 25 vs. 27 , respectively) and the logarithmic transformation is more widely accepted than the "inverse square root". The value of $\lambda$ chosen for the Aranda-Ordaz transformation was 0.7 and $\lambda$ was taken as 0.5 for the folded power transformation.

With these three transformations plus the angular, the square root and the untransformed data, we proceeded to perform the corresponding linear regression analyses of viability onto initial density for all mono- and duocultures. The slopes can be used in order to determine the response of each strain under competition. The regression coefficients obtained using the different transformations cannot be compared directly because of the changes of scale involved. However, we can compare the corresponding levels of significance, both for the linear regression (with an analysis of variance) and for the differences between slopes and ordinates at the origin (with corresponding analyses of covariance). This approach will indicate whether a certain transformation behaves markedly differently from the rest and whether the linear model is valid or not for it.

The Aranda-Ordaz and the folded power transformations were always coincident in their levels of significance both for the linear model and for differences between slopes and ordinates at the origin. Of the 42 regression analyses (10 monocultures, 16 duocultures), the untransformed data 


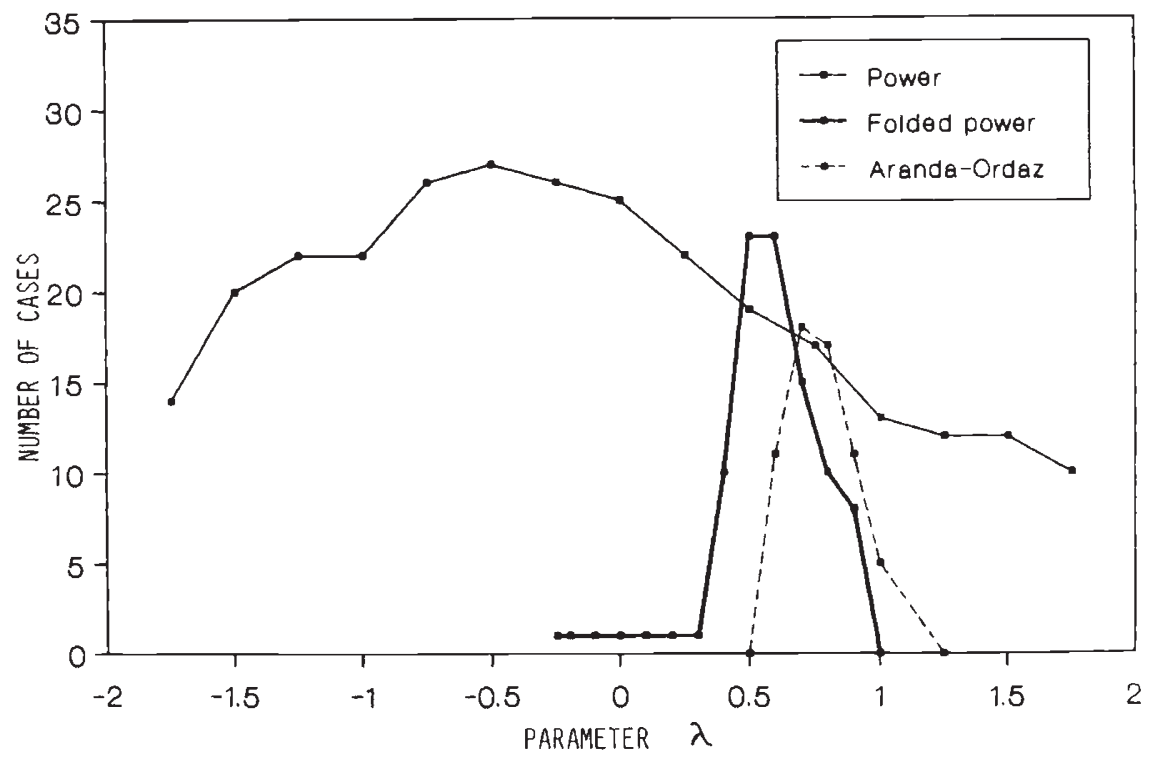

Figure 1 Plots of the number of cases (out of 42) in which a given value of $\lambda$ belongs to the 0.95 confidence interval for the power, folded power and Aranda-Ordaz transformations.

give different levels of significance when compared to the rest of the transformations in six cases, the square-root in four cases, and the logarithmic and the angular transformations in two cases each (table 1(a)). Of the 32 comparisons between slopes and between ordinates, in three cases the results with the untransformed data differed from the rest. The logarithmic transformation was not coincident with the rest in two cases and the square-root in one (table 1(b)).

The above results indicate that either the Aranda-Ordaz or the folded power transformation would be preferable, as they maximize the requirements of normality, additivity and homoscedasticity, and do not differ from the rest in the comparisons of levels of significance. We

Table 1 Summary of discrepancies in the levels of significance between the various transformations for the regression analyses (a) and the analyses of covariance (b). The most frequent levels of significance correspond to the Aranda-Ordaz (AO) and folded power transformations

\section{Transformation}

\begin{tabular}{|c|c|c|c|c|c|c|}
\hline $\begin{array}{l}\text { Indicator } \\
\text { strain }\end{array}$ & $\begin{array}{l}\text { Associate } \\
\text { strain }\end{array}$ & None & Square-root & Logarithmic & Angular & $\mathrm{AO}$ \\
\hline \multicolumn{7}{|c|}{ (a) Regression analyses } \\
\hline wild & se & & * & & & $* *$ \\
\hline 2.74.B & wild & $* * *$ & & & & $* *$ \\
\hline$w$ & wild & $*$ & & $*$ & & $* *$ \\
\hline Or-R & se & * & & & & ns \\
\hline Or-R & bo & $*$ & $* * *$ & & $* * *$ & $* *$ \\
\hline$s e^{790}$ & Or-R & ns & $* *$ & ns & & $*$ \\
\hline se & Or-R & $*$ & ns & & * & $* *$ \\
\hline \multicolumn{7}{|c|}{ (b) Analyses of covariance } \\
\hline wild & $c d^{770}$ & $* * *$ & & $* * *$ & & $* *$ \\
\hline 1.51 .3 & wild & $* * *$ & & $* * *$ & & $* *$ \\
\hline Or-R & $v$ & $* * *$ & & & & $* *$ \\
\hline Or-R & 1.51 .3 & & $* *$ & & & $* * *$ \\
\hline
\end{tabular}

ns $=$ not significant; $*=P \leqq 0.05 ; * *=P<0.01 ; * * *=P<0.001$. 
have decided to present only the results obtained with the latter since the differences between them $\left(10^{-3}-10^{-4}\right)$ are so small.

For both monocultures and duocultures, the significance of the regression in an analysis of variance was calculated. Of the monocultures, only that for the wild strain yielded a slope that did not differ significantly from zero. This indicates that, at least in the interval considered, wild viability is independent of initial density. Hence, no intragenotypic interactions are detected. The other nine strains yielded highly significant, negative slopes of the kind to be expected with increasing initial densities. Most of the slopes in duoculture were highly significant ( 26 out of 32 cases), with more negative (16) than positive signs (10).

According to the analysis of Mather and Caligari (1981), monoculture and duoculture slopes are compared by means of analyses of covariance (table 2). When the two slopes are significantly different, the nature of the competition can be determined by comparison of their values according to the following scheme:

$$
\begin{aligned}
& b_{\mathrm{d}}<0\left\{\begin{array}{l}
b_{\mathrm{d}}<b_{\mathrm{m}} \cdots \text { facilitation } \\
b_{\mathrm{d}}>b_{\mathrm{m}} \cdots \text { competition }
\end{array}\right. \\
& b_{\mathrm{d}}=0 \cdots \text { intragenotypic }=\text { intergenotypic } \\
& b_{\mathrm{d}}>0 \cdots \text { hypercompetition }
\end{aligned}
$$

where $b_{\mathrm{m}}$ and $b_{\mathrm{d}}$ represent the monoculture and duoculture slopes, respectively. When the two slopes do not differ significantly, the substitution of individuals of the indicator strain by those of the associate strain has no effect on the indicator

Table 2 Summaries of the analyses of covariance comparing mono- and duoculture slopes for all combinations of indicator and associate strains. The nature of the competitive

\begin{tabular}{|c|c|c|c|c|}
\hline $\begin{array}{l}\text { Indicator } \\
\text { strain }\end{array}$ & $\begin{array}{l}\text { Associate } \\
\text { strain }\end{array}$ & $\begin{array}{l}\text { Competitive } \\
\text { response }\end{array}$ & d.f. & $\mathrm{F}$ \\
\hline wild & $c d^{77 \circ}$ & $\mathrm{H}$ & 1,276 & $10 \cdot 22^{* *}$ \\
\hline wild & $s e^{790}$ & $\mathrm{~F}$ & 1,275 & $37 \cdot 13^{* * *}$ \\
\hline wild & 2.74.B & $\mathrm{F}$ & 1,258 & $40 \cdot 32^{* * *}$ \\
\hline wild & 1.51 .3 & $\mathrm{~F}$ & 1,276 & $29.70^{* * *}$ \\
\hline wild & se & $\mathrm{I}, \mathrm{E}$ & 1,276 & $2 \cdot 56$ \\
\hline wild & bo & $\mathrm{H}$ & 1,276 & $55 \cdot 66^{* * *}$ \\
\hline wild & $v$ & $\mathrm{~F}$ & 1,276 & $60 \cdot 48^{* * *}$ \\
\hline wild & $w$ & $\mathrm{~F}$ & 1,276 & $15 \cdot 98 * * *$ \\
\hline $\mathrm{cd}^{770}$ & wild & $\mathrm{C}$ & 1,276 & $23 \cdot 35^{* * *}$ \\
\hline$s e^{790}$ & wild & $\mathrm{H}$ & 1,247 & $88 \cdot 09 * * *$ \\
\hline 2.74.B & wild & I & 1,198 & 1.89 \\
\hline 1.51 .3 & wild & $\mathrm{C}$ & 1,274 & $8 \cdot 80^{* *}$ \\
\hline se & wild & $\mathrm{E}$ & 1,274 & $151 \cdot 76^{* * *}$ \\
\hline bo & wild & I & 1,276 & 1.96 \\
\hline$v$ & wild & $\mathrm{H}$ & 1,276 & $240 \cdot 32^{* *}$ \\
\hline$w$ & wild & $\mathrm{H}$ & 1,230 & $23 \cdot 39 * * *$ \\
\hline Or-R & $c d^{770}$ & $\mathrm{C}$ & 1,276 & $35 \cdot 35^{* * *}$ \\
\hline Or-R & $s e^{790}$ & $\mathrm{C}$ & 1,273 & $38.91^{* * *}$ \\
\hline Or-R & 2.74.B & $\mathrm{E}$ & 1,218 & $33 \cdot 52^{* * *}$ \\
\hline Or-R & 1.51 .3 & $\mathrm{C}$ & 1,276 & $14 \cdot 53^{* * *}$ \\
\hline Or-R & se & $\mathrm{E}$ & 1,276 & $80 \cdot 52^{* * *}$ \\
\hline Or-R & bo & $\mathrm{H}$ & 1,276 & $167 \cdot 86^{* * *}$ \\
\hline Or-R & $v$ & $\mathrm{C}$ & 1,276 & $10 \cdot 38^{* *}$ \\
\hline Or-R & $w$ & $\mathrm{E}$ & 1,267 & $69 \cdot 11^{\text {*** }}$ \\
\hline$c d^{770}$ & Or-R & $\mathrm{H}$ & 1,276 & $299 \cdot 17^{* * *}$ \\
\hline$s e^{790}$ & Or-R & $\mathrm{H}$ & 1,245 & $31 \cdot 64^{* * *}$ \\
\hline 2.74.B & Or-R & $\mathrm{H}$ & 1,160 & $56 \cdot 43 * * *$ \\
\hline 1.51 .3 & Or-R & $\mathrm{E}$ & 1,274 & $84 \cdot 49 * * *$ \\
\hline se & Or-R & $\mathrm{C}$ & 1,274 & $52 \cdot 17^{* * *}$ \\
\hline bo & Or-R & $\mathrm{C}$ & 1,276 & $15 \cdot 46^{* * *}$ \\
\hline$v$ & Or-R & $\mathrm{H}$ & 1,276 & $397 \cdot 05^{* * *}$ \\
\hline$w$ & Or-R & $\mathrm{E}$ & 1,221 & $6.29^{*}$ \\
\hline
\end{tabular}
response in each case (see text) is indicated $(\mathrm{C}=$ competition, $\mathrm{H}=$ hypercompetition, $\mathrm{F}=$ facilitation, $\mathrm{I}=$ indifference, and $\mathrm{E}=$ equal intra- and intergenotypic effects)

${ }^{*}=P \leqq 0.05 ;{ }^{* *}=P<0.01 ;{ }^{* *}=P<0.001$. 
viability. This situation may be classified as "indifference".

These comparisons are shown in table 2 and it is evident that all but two of the reciprocal duocultures behave differently. The exceptions are se/wild (I, E); wild/se (E) and w/Or-R (E); Or$\mathrm{R} / w(\mathrm{E})$. However, these comparisons necessarily take into account the effect of the corresponding monocultures (intragenotypic effects) via the comparisons between mono- and duoculture slopes. We are interested in comparing the intergenotypic effects of different strains when competing with the same competitor. The intra- and intergenotypic effects can be separated by subtracting the monoculture and duoculture slopes $\left(b_{\mathrm{m}}-b_{\mathrm{d}}\right)$ and the resulting intergenotypic competitive effects for all the strains are compared in table 3 . There is only one case in which the intergenotypic effects of both wild type strains on a mutant one $(v)$ are not different. It can be observed that the wild strain suffers much less intergenotypic competition than Or-R, with most cases being facilitation $\left[\left(b_{\mathrm{m}}-\right.\right.$ $\left.\left.b_{\mathrm{d}}\right)>0\right]$ rather than competition $\left[\left(b_{\mathrm{m}}-b_{\mathrm{d}}\right)<0\right]$. This is presumably related to the absence of detectable intragenotypic competition in the wild strain.

\section{DISCUSSION}

Despite the necessity to fulfil various requirements for the correct application of least squares analyses to viability data, we have not detected many significant differences when applying these tests to untransformed or differently transformed data. Of the 444 analyses of regression and covariance involving five different transformations as well as untransformed data, only 20 showed discrepancies. Most of these were merely in the level of significance and nine of them correspond to the untransformed data (table 1).
These results do not imply that transformations are unnecessary and superfluous. Rather, they imply that almost any normalizing transformation will be perfectly valid for the analysis of viability data. In this case we have used the folded power transformation $\left(y^{\prime}=\sqrt{y}-\sqrt{(1-y)}\right)$ for the remaining analyses. However, the angular transformation behaves almost like it and it is used more often in the literature, making it a good choice for this kind of analysis.

Most of the strains analysed show intragenotypic interactions. The only strain which does not is wild and the original data show that its viability remains constant at around 0.41 $( \pm 0.03)$ for all the densities tested. This figure is lower than the viabilities in monocultures of the remaining strains at low densities. Castro et al. (1985) showed that the wild strain had a higher optimal density (initial density with the highest survival rate) than the strains $c d^{770}$ and se, also used in this experiment. If the initial densities used do not include the point of optimal density, it may be difficult to detect intragenotypic effects (Wallace, 1981, chapter 16).

Those monocultures with lower intrinsic mortality rates (high viability at low initial densities) show higher intragenotypic effects and vice versa. Losses due to intrinsic mortality are densityindependent and it can be assumed that they occur in the early stages of development. Hence, these dead individuals do not enter the competing populations, which implies lower effective initial densities. The absence of intragenotypic effects already mentioned for wild is quite well explained by this fact and its higher optimal density (Castro et al., 1985).

The absence of intragenotypic competition for the wild strain means that associate competitors may only be classified as displaying hypercompetition, facilitation or indifference, depending on

Table 3 Intergenotypic effects of each competitor in the duocultures. The first column indicates the effect of the mutant strains on the wild type ones. The second column indicates the effect of the wild type strain on the mutant. The two values indicated by $(*)$ do not differ significantly, and those marked with (ns) are not significantly different from 0

\begin{tabular}{|c|c|c|c|c|}
\hline \multirow[b]{2}{*}{ Mutant } & \multicolumn{4}{|l|}{ Wild type } \\
\hline & Wild & & Or-R & \\
\hline$c d^{770}$ & -0.0034 & -0.0053 & -0.0088 & -0.0182 \\
\hline$s e^{790}$ & 0.0068 & -0.0142 & -0.0088 & -0.0105 \\
\hline $2.74 . \mathrm{B}$ & 0.0079 & $-0.0022^{\mathrm{ns}}$ & -0.0158 & -0.0141 \\
\hline 1.51 .3 & 0.0068 & -0.0037 & -0.0057 & -0.0115 \\
\hline se & $0.0017^{\mathrm{ns}}$ & -0.0144 & -0.0126 & -0.0106 \\
\hline bo & -0.0081 & $0.0018^{\text {ns }}$ & -0.0166 & -0.0052 \\
\hline$v$ & 0.0091 & $-0.0175^{*}$ & -0.0048 & $-0.0184^{*}$ \\
\hline$w$ & 0.0045 & -0.0071 & -0.0124 & -0.0043 \\
\hline
\end{tabular}


whether the duoculture slope is higher, lower or equal to 0 . In such cases, it might be better to analyse solely intergenotypic effects instead of comparing them with the non-detectable intragenotypic ones. The experiments of Castro et al. (1986) with preconditioned media using the strains wild, $c d^{770}$ and se provide a good explanation for the reciprocal effects of wild and $s e$, but not for those of wild and $c d^{770}$. The two first strains have very similar effects (uric acid released, food ingested and free water in the medium). This implies that intra- and intergenotypic effects are similar, which can be seen in table 2 . It should be noted that as intragenotypic effects cannot be detected for the wild strain, indifference (I) and equality (E) of intra and intergenotypic effects cannot be distinguished. Similarly, the results of Castro et al. (1986) explain the effect of wild on $c d^{770}$ larvae, but cannot account for the reciprocal ones. Nevertheless, the intergenotypic effects of $c d^{770}$ on wild, although statistically significant, are not very intense (table 3 ). The remaining interactions have not been analysed with this experimental design, and an explanation for the observed responses in physical-chemical terms remains unknown. In any case, competitive interactions are the result of a complex array of biological phenomena (feeding rates, food conversion efficiencies, critical weights, postcritical weight feeding and interference or facilitation through media conditioning, among others). Most of these phenomena have been shown to play an important role in determining whether a larva will survive or not to adulthood (Burnet et al., 1977; Nunney, 1983; Joshi and Mueller, 1988; Mueller, 1990; Mueller et al., 1990).

The results of duocultures are very diverse. Generally, Oregon-R suffers stronger intergenotypic effects when cultured with mutants than wild, and Or-R also exerts stronger effects than wild. As we have mentioned already, this is expected in part from the behaviour of wild monocultures. However, any attempt to hypothesize about the outcome of duocultures from previous information of monocultures is doomed to fail (Bakker, 1961; Mather and Caligari, 1981). In these experiments wild seems generally to be a worse competitor than Oregon- $\mathrm{R}$. However, this is not true in all cases, as can be seen by the effects of wild on $s e^{790}$, se and $v$ (table 3 ).

A clear conclusion can be drawn from these results: there is no way of comparing two strains with identical phenotype by means of using a third, marker strain. There is no consistency in the results of duocultures when we compare any mutant strain and both wild type ones. This makes it impossible to decide which strain, if any, should be chosen in order to compare wild and Oregon- $\mathrm{R}$ (table 3 ).

Finally, on the strength of these results we may suggest a similar rule to the one proposed originally by Bakker (1961). If the outcome of duocultures cannot be predicted from the corresponding monocultures, similarly the interactions of two undistinguishable genotypes cannot be predicted by comparisons involving a third one.

Acknowledgements We thank J. J. Tarín for his technical help. This research has been supported by grants from Conselleria Valenciana de Educació y Ciència (J.C.A.), M.E.C. Programa General de Extranjero y Programa de Reincorporación de Doctores y Tecnólogos (F.G.C.), Institució Valenciana d'Estudis i Investigació (F.G.C. and A.M.) and DGICYT PB860517 (A.M.). The authors are grateful to two anonymous reviewers for their comments and suggestions.

\section{REFERENCES}

ANSCOMBE, F. J. 1948. The transformation of Poisson, binomial and negative binomial data. Biometrika, 35, 246-254.

ARANDA-ORDAZ, F. J. 1981. On two families of transformations to additivity for binary response data. Biometrika, $68,357-$ 363.

Atkinson, A. C. 1985. Plots, Transformations and Regression. Oxford University Press, Oxford.

BAKKER, K. 1961. An analysis of factors which determine success in competition for food among larvae of Drosophila melanogaster. Arch. Néerland Zool., 14, 200-281.

BOX, G. E. P. AND COX, D. R. 1964. An analysis of transformations (with discussion). J. R. Statist. Soc. B, 26, 211-246.

BURNET, B., SEWELL, D. AND BOS, M. 1977. Genetic analysis of larval feeding behavior in Drosophila melanogaster. II. Growth relations and competition between selected lines. Genet. Res., Camb., 30, 149-161.

CALIGARI, P. D. S. 1980. Competitive interactions in Drosophila melanogaster. I. Monocultures. Heredity, 45, 219-231.

CALIGARI, P. D. S. AND MATHER, K. 1984. Competitive interactions in Drosophila melanogaster. III. Triocultures. Heredity, 52, 255-264.

CALIGARI, P. D. S. AND MATHER, K. 1988. Competitive interactions in Drosophila melanogaster. IV. Chromosome assay. Heredity, 60, 355-366.

CASTRO, J. A., MOYA, A. AND MENSUA, J. L. 1985. Competitive selection in mono-, di- and tri-genotype cultures of Drosophila melanogaster. Z. Zool. Syst. Evolut.-forsch, 23, 214-228.

CASTRO, J. A., BOTELlA, L. M. AND MENSUA, J. L. 1986. Effect of conditioned media on three genotypes of Drosophila melanogaster: physical, chemical and biological aspects. Arch. Insect Biochem. Physiol,, 3, 485-497.

ECONOMOS, A. C., LINTS, C. V. AND LINTS, F. A. 1982. On the mechanisms of the effects of larval density and temperature on Drosophila development. In Lakovaara, S. (ed.) Advances in Genetics, Development and Evolution of Drosophila, Plenum Press, New York, pp. 149-164.

EGGLESTON, P. 1987. The use of genetic markers in the analysis of competitive interactions in Drosophila melanogaster. Genetica, 72, 181-186.

JOSHI, A. AND MUELLER, L. D. 1988. Evolution of higher feeding rate in Drosophila due to density-dependent natural selection. Evolution, 42, 1090-1093. 
KELLEY, S. E. 1985. The effects of neighbors as environments: characterization of the competitive performance of Danthonia spicata genotypes. In Jacquard, P. et al. (eds) Genetic Differentiation and Dispersal in Plants, Springer-Verlag, Berlin, pp. 203-221.

KELLEY, S. E. AND CLAY, K. 1987. Interspecific competitive interactions and the maintenance of genotypic variation within two perennial grasses. Evolution, 41, 92-103.

MATHER, K. AND CALIGARI, P. D. S. 1981. Competitive interactions in Drosophila melanogaster. II. Measurement of competition. Heredity, 46, 239-254.

MATHER, K. AND CAligari, P. D. S. 1983. Pressure and response in competitive interactions. Heredity, 51, 435-454.

MATHER, K., HILL, J. AND CALIGARI, P. D. S. 1982. Analysis of competitive ability among genotypes of perennial ryegrass. Heredity, 48, 421-434.

MOLINA, V., GONZALEZ-CANDELAS, F. AND MENSUA, J. L. 1988. Relationship between density-and frequency-dependent effects on two strains of Drosophila melanogaster. Gén. Sél. Evol., 20, 425-434.
Mosteller, F. ANd tUkey, J. W. 1977. Data Analysis and Regression. Addison-Wesley, Reading.

MOYA, A. AND BOTELLA, L. M. 1985. Larval-to-adult and pupato-adult mortality dynamics in crowded cultures of Drosophila melanogaster. Genetica, 67, 201-207.

MOYA, A. AND CASTRO, J. A. 1986. Larval competition in Drosophila melanogaster: the model of the bands of density. Oikos, 47, 280-286.

MUELLER, L. D. 1990. Density-dependent natural selection does not increase efficiency. Evol. Ecol. (In press).

MUELLER, L. D., GONZALEZ-CANDELAS, F. AND SWEET, V. F. 1990. Components of density-dependent population dynamics: models and tests with Drosophila. Amer. Natur. (In press).

NUNNEY, L. 1983. Sex differences in larval competition in Drosophila melanogaster: the testing of a competition model and its relevance to frequency dependent selection. Amer. Natur., 121, 67-93.

WAllace, B. 1981. Basic Population Genetics. Columbia University Press, New York. 